\title{
DEVELOPMENT OF OPTO-PNEUMATIC ON-OFF VALVE
}

\author{
Tetsuya AKAGI*, Shujiro DOHTA**, Hisashi MATSUSHITA** and Koichi TAKECHI** \\ * Department of Electronics and Control Engineering, Tsuyama National College of Technology \\ 624-1, Numa, Tsuyama, Okayama, 708-8509 Japan \\ (E-mail: akagi@tsuyama-ct.ac.jp) \\ ** Department of Assistive and Rehabilitation Engineering, Okayama University of Science \\ 1-1, Ridai-cho, Okayama, 700-0005 Japan
}

\begin{abstract}
An optical servo system is a new control system that can be used in hazardous environments. The purpose of our study is to develop such an optical control system. In a previous study, we had realized an optical control system that executed cart positioning using optical control signals instead of electric signals. We developed an optical servo valve in which the output pressure was proportional to input optical power. As a next step, we needed to develop another type of optical valve in order to get higher-pressure gain. In this study, we propose and produce an optical on-off valve that consists of an optical on-off device and a fluid amplifier, and the structure, operating principle and fundamental characteristics of the valve are investigated. As the result, we obtained a higher output pressure of the tested valve compared with the previous one. And we proposed the analytical model of the optical on-off device and identified the system parameters. We confirmed their validity by comparing them with experimental results. And finally, we improved the dynamics of the device by using a feedback passage plate based on analytical results of the device.
\end{abstract}

\section{KEY WORDS}

Optical Servo System, Opto-Pneumatic On-Off Valve, Photo-Fluidic Interface, Laminar Proportional Amplifier

\section{NOMENCLATURES}

$C d$ : discharge coefficient of nozzle or orifice

$K u$ : empirical constant which relates the area to input optical power $\left[\mathrm{m}^{2} / \mathrm{mW}\right]$

$K f$ : empirical constant which relates the area to pressure of control port in feedback LPAs $\left[\mathrm{m}^{2} / \mathrm{Pa}\right]$

$O u$ : input optical power [mW]

$P$ : absolute pressure $[\mathrm{Pa}]$

$P a t:$ atmospheric pressure $[\mathrm{Pa}]$

$Q$ : mass flow rate $[\mathrm{kg} / \mathrm{s}]$

$R$ : gas constant $[\mathrm{J} /(\mathrm{kg} \cdot \mathrm{K})]$

$S$ : area of opening of interface and LPA $\left[\mathrm{m}^{2}\right]$

$T$ : absolute temperature [K]
$V \quad$ : chamber volume $\left[\mathrm{m}^{3}\right]$

$\rho:$ density of air $\left[\mathrm{kg} / \mathrm{m}^{3}\right]$

$\kappa:$ specific heat ratio of air $(=1.4)$

In symbols with a subscript, the first subscript ( $i$ or $f$ ) indicates the element, i.e., the interface, feedback LPAs. The 2 nd subscript ( $c, n, o$ or $s$ ) indicates the position, i.e., the control port, vent, outlet or supply. The 3rd subscript ( $R$ or $L$ ) indicates the center, right or left side. The last subscript 0 indicates the initial value of each quantity.

\section{INTRODUCTION}

An optical servo system proposed by Nakada et al.[1] is a novel control system in which a part or all of the 
electric or electronic components of the existing control system are replaced with optical components in order to exceed the performance of the existing electronic servo system which suffers from electromagnetic noise. The purpose of our study is to develop an optical control system to be used in hazardous environments[2]. To construct the optical servo system, first, we developed an optical servo valve which consists of a photo-fluidic interface and 2-stage fluid booster amplifiers driven by very low differential pressure from the photo-fluidic interface[3]. Then, we developed two types of cart position control systems in which the optical control signal is fed back directly or indirectly[4]. We extended to the optical servo system at a larger scale by improving the optical servo valve[5]. And we investigated the optimal design of the optical servo valve in order to improve the control performance by using a proposed analytical model of the whole control system including the optical servo valve[7][8]. In the next step, we needed to design and develop an opto-pneumatic on-off valve that could be driven by lower optical power in order to apply the optical servo system into a more flammable environment.

In this paper, we aim to develop the opto-pneumatic on-off valve. First, we describe the construction of the valve: an optical on-off device that consists of a photofluidic interface and feedback laminar proportional amplifiers (LPA for short) and a fluid amplifier driven by a low pressure. Next, we investigate static and dynamic characteristics of the tested valve. We produce an analytical model of optical on-off device in order to improve the performance of the device. Finally, we confirm the validity of the model by comparing with the experimental results of the device.

\section{CONSTRUCTION AND OPERATING PRINCIPLE}

Figure 1 shows the construction of an opto-pneumatic on-off valve. The valve consists of an optical on-off device that can realize switching on or off the output pressure for optical input and a fluid amplifier that can be driven by low differential pressure. The detailed construction and operating principle of each element will be described in the following section.

\section{Optical on-off device}

Figure 2 shows construction of the optical on-off device. The device consists of photo-fluidic interface as shown in Fig.2 (a), feedback LPAs which consists of LPA, feedback flow passage, check valve and offset adjusting valve as shown in Fig.2 (b). The size of the photo-fluidic interface and feedback LPAs are $34 \times 30$ $\mathrm{mm}$. The operating principle of the device is as follows: In the initial condition of feedback LPAs, an initial offset of output differential pressure and flow rate from photo-fluidic interface is given by using two adjusting valves. This offset pressure is carried on a control port (inlet) and deflects a nozzle flow to one outlet, it makes

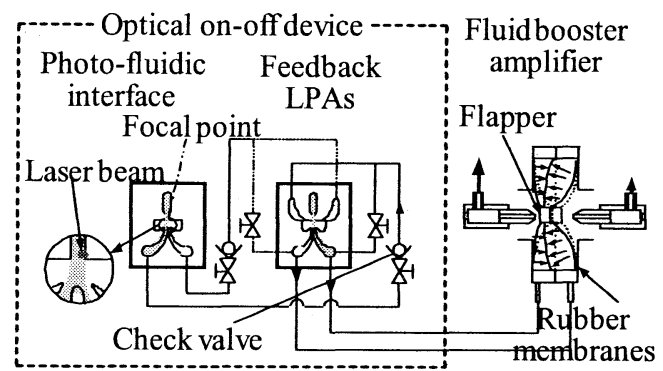

Fig.1 Construction of opto-pneumatic on-off valve

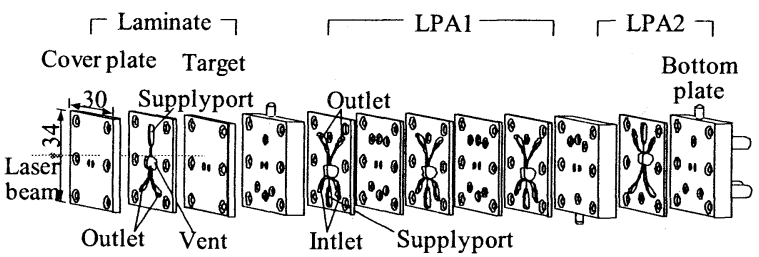

(a) Construction of photo-fluidic interface

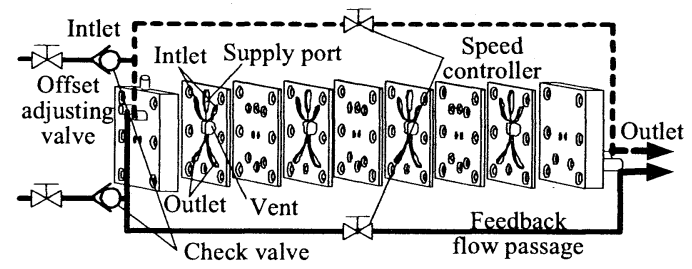

(b) Construction of feedback LPAs

Fig.2 Construction of optical on-off device

a differential pressure and flow rate between the two outlets of the LPAs. The generated flow is fed back to the crossing control port of the LPA through the feedback flow passage. It creates a higher differential pressure between the two outlets. An optical signal (laser beam) inputs to the optical on-off device. In the photo-fluidic interface, the laser beam is applied through the transparent cover plate to the nozzle edge. The light energy of the laser beam is converted to thermal energy on the layer of carbon black, and the temperature of the target rises. Consequently, the temperature of the fluid around the laser focal point rises and fluid viscosity is changed. This change makes the nozzle flow asymmetric with respect to the nozzle axis, and the asymmetry causes a difference in output pressure and flow rate. This differential pressure is amplified by an LPA in the interface[3]. This pressure makes the nozzle flow deflect to another outlet of feedback LPAs. The output differential pressure between the two outlets can be reversed and amplified by a fluid amplifier. Then, we can adjust the feedback flow rate by using a speed controller. check valves automatically prevent a reversed flow from feedback LPAs to the photo-fluidic interface. Fluid amplifier

The structure of the tested booster amplifier is shown in Fig.3. The amplifier consists of a double acting type flapper membrane and a nozzle-flapper valve. The 
operating principle of the amplifier is as follows: When the low differential pressure from the optical on-off device is applied to the two chambers in the fluid amplifier through two inlets, the rubber membranes are deformed and the central flapper moves. The displacement of the flapper makes the backpressure change and the amplified output fluid power can be obtained. In our previous study[4]-[8], a 2-stage booster amplifier was used to obtain the fluid power needed to drive a pneumatic cylinder because of lack of output fluid power from the photo-fluidic interface. By using the tested optical on-off device and fluid amplifier, we can obtain output pressure that is enough to drive pneumatic actuators.

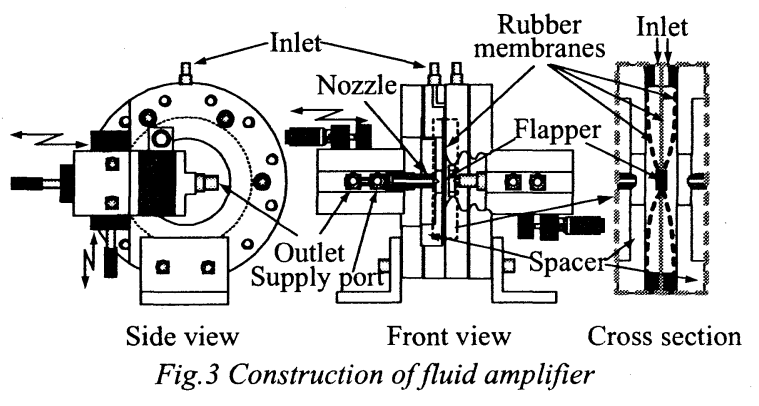

\section{CHARACTERISTICS OF OPTICAL ON-OFF DEVICE}

\section{Static characteristics}

Figure 4 shows relations between input optical power and output differential pressure of the photo-fluidic interface and the optical on-off device. In Fig.4, each symbol $\square$ and $\triangle$ shows output pressure by using the optical on-off device, the device without feedback flow passages and photo-fluidic interface, respectively. In the experiment, supply pressure of feedback LPAs is $40 \mathrm{kPa}$. From Fig.4, we can find that switching optical power of the device, such as on and off actions, are differences. In increasing input optical power from $0 \mathrm{~mW}$ to $40 \mathrm{~mW}$, the device switches on at the point of $20 \mathrm{~mW}$. In decreasing optical power from $40 \mathrm{~mW}$ to $0 \mathrm{~mW}$, the device switches off at $10 \mathrm{~mW}$. It can be found that the device generated differential pressure of $\pm 14 \mathrm{kPa}$ for

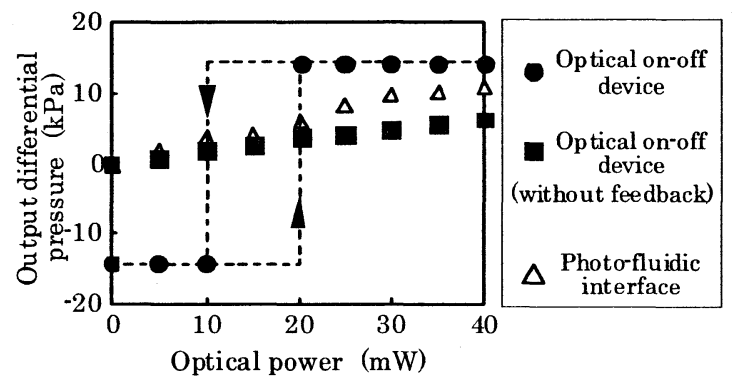

Fig.4 Relation between optical power and output pressure of optical on-off device.

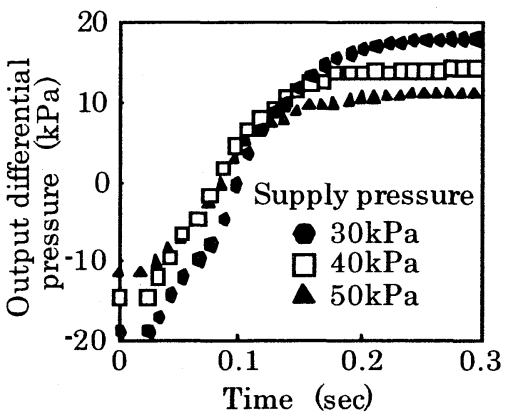

(a) Switched on

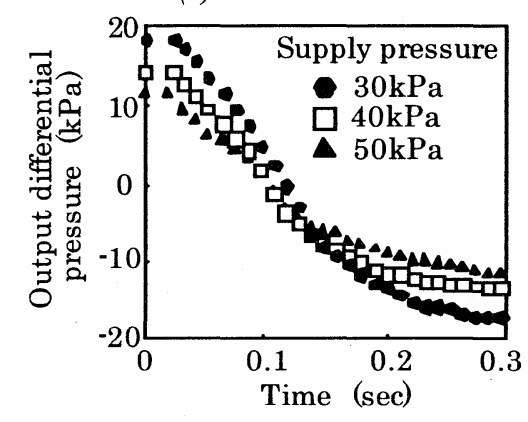

(b) Switched off

Fig.5 Step response of optical on-off device

input optical power of $20 \mathrm{~mW}$. The generated differential pressure of the device is 9.3 times as much as that of a photo-fluidic interface with an output pressure of $3 \mathrm{kPa}$. Compared with the performance using the device without feedback flow passages, it generated 4.7 times the output pressure. We can confirm the validity of using feedback passages in order to get higher converter gain from an optical signal to output pressure.

\section{Dynamic characteristics}

Figure 5 shows the transient response of output pressure of the photo-fluidic interface and the optical onoff device. Figure 5 (a) shows response when the stepwise optical power of $25 \mathrm{~mW}$ is applied to the device. Figure 5 (b) shows the results when the applied optical power of $25 \mathrm{~mW}$ turns to $0 \mathrm{~mW}$. In Fig.5, each symbol $\bigcirc$ and $\boldsymbol{\Delta}$ shows transient response of output pressure when supply pressure of feedback LPAs is 30 , 40 and $50 \mathrm{kPa}$, respectively. We estimated the dynamics of the device by using the sum of a dead time and a time constant as a response time. In the case of using a supply pressure of $40 \mathrm{kPa}$, it can be seen that response time when the device switches on is $0.098 \mathrm{~s}$, and the response time when the device switches off is $0.115 \mathrm{~s}$.

\section{CHARACTERISTICS OF TESTED ON-OFF VALVE}

\section{Static characteristics}

Figure 6 shows the relation between input optical power and output differential pressure of the opto- 


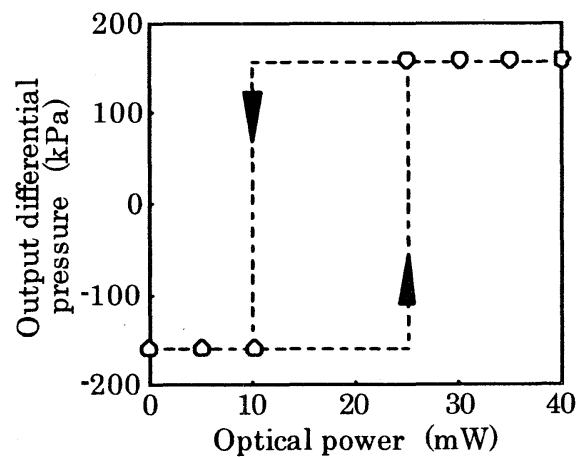

Fig.6 Relation between optical power and output pressure of opto-pneumatic on-off valve

pneumatic on-off valve. When the input optical power increases, output differential pressure of the valve switches from $-155 \mathrm{kPa}$ to $155 \mathrm{kPa}$ at the point of 25 $\mathrm{mW}$. When the input optical power decreases from 40 $\mathrm{mW}$ to $0 \mathrm{~mW}$, output pressure changes the reverse at the point of input optical power of $10 \mathrm{~mW}$. Comparing the output pressure of the valve with that of the previous optical servo valve [6], maximum output pressure of the valve can be improved 4.5 times.

\section{Dynamic characteristics}

In order to estimate the dynamics of the tested valve, Figure 7 shows transient responses of the tested valve connected with a volume of $5 \mathrm{cc}$ for stepwise input optical signal. In Fig.7, each symbol and $\triangle$ shows a transient response of output pressure when the optical signal switches on and off into the valve, respectively. It can be seen that response time which is the sum of a dead time and a time constant is $0.137 \mathrm{~s}$ (switching on) or $0.146 \mathrm{~s}$ (switching off). The dead time in the response depends on the time required to deform the flapper membrane so as to open one side of the nozzle that is closed by the flapper. We think that it is possible to improve the dynamics of the valve by using a suitable arrangement between the two nozzles and a flapper in the amplifier.

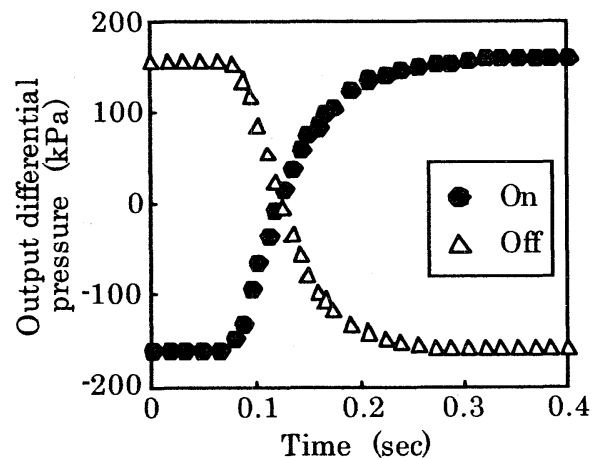

Fig.7 Step response of opto-pneumatic on-off valve

\section{ANALYSIS OF OPTICAL ON-OFF DEVICE}

In order to estimate and improve the performance of the optical on-off device, we proposed the analytical model of the device as shown in Fig. 8. The model is divided into two parts; one is a photo-fluidic interface, the other is feedback LPAs. In the photo-fluidic interface, the relation between input optical power $\mathrm{Ou}$ and area of opening $S i$ is given by

$$
\begin{array}{lll}
S_{i j}=S_{i j 0}-K u & \cdot O u & (j=s L, n R) \\
S_{i k}=S_{i k 0}+K u & \cdot O u & (k=s R, n L)
\end{array}
$$

where $K u$ is an empirical constant which relates the area $S i$ to input optical power $O u$. As a flow in the photofluidic interface is incompressible [3], mass flow rate Qioj in the outlet of the interface can be described by

$$
Q_{i o j}=S_{i s j} \sqrt{2 \rho\left(P_{i s}-P_{i o j}\right)}-S_{i n j} \sqrt{2 \rho\left(P_{i o j}-P_{a t}\right)} \quad(j=R, L)
$$

Mass flow rate Qiofj into the inlet of the feedback LPA from photo-fluidic interface can be described by

$$
Q_{i o f j}=C d_{f s} \times S_{i o f j} \sqrt{2 \rho\left(P_{i o j}-P_{k}\right)} \quad(j=R, L \quad k=L, R)
$$

From the characteristic of the check valve:

$$
\text { If } P_{i o j}-P_{k}<\Delta P_{d j} \text { then } Q_{i o f j}=0 \quad(j=R, L \quad k=L, R)
$$

Assuming the change of state for gas in the interface

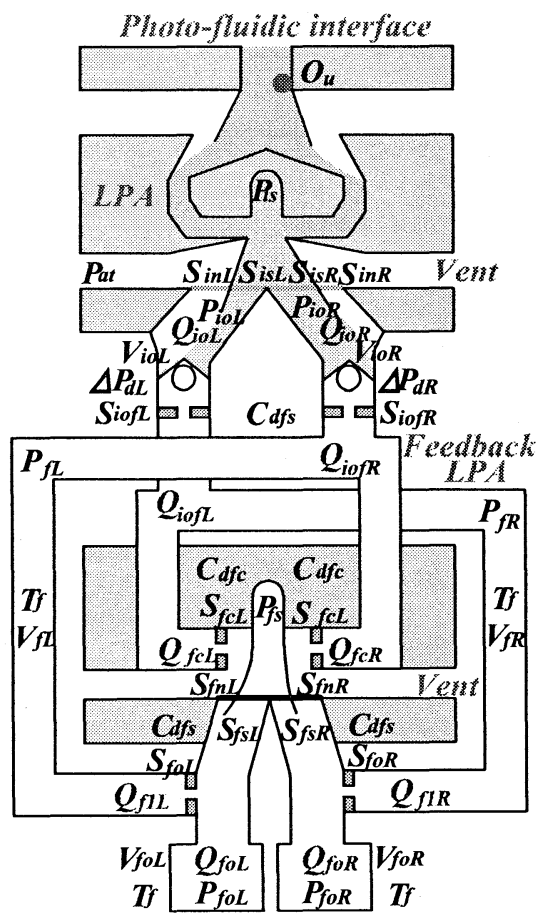

Fig.8 Analytical model of optical on-off device 
chamber is adiabatic, the following expression is derived from the law of energy conservation

$$
\frac{d P_{i o j}}{d t}=\frac{\kappa R T_{f}}{V_{i o j}}\left(Q_{i o j}-Q_{i o f j}\right) \quad \underset{(j=R, L)}{ }
$$

Mass flow rate $Q f l j$ into the inlet of the feedback LPA from outlet of feedback LPA and pressure $P f j$ can be described by

$$
\begin{gathered}
Q_{f l j}=C d_{f s} \times S_{f o j} \sqrt{2 \rho\left(P_{f o j}-P_{f j}\right)} \quad(j=R, L) \\
\frac{d P_{f j}}{d t}=\frac{\kappa R T_{f}}{V_{f j}}\left(Q_{f l j}+Q_{i o f k}-Q_{f c k}\right){ }_{(j=R, L \quad k=L, R)}
\end{gathered}
$$

Mass flow rate $Q_{f c j}$ into the control port of the feedback passage from photo-fluidic interface can be described by

$$
Q_{f c j}=C d_{f c} \times S_{f c j} \sqrt{2 \rho\left(P_{f k}-P_{a t}\right)} \quad(j=R, L \quad k=L, R)
$$

Area of opening $S f t$ and $S f k$ in feedback LPAs and vent are given by the following equations

$$
\begin{gathered}
\Delta P_{f}=\frac{P_{f k}-P_{f j}}{P_{f S}-P_{a t}} \quad(j=R, L \quad k=L, R) \\
S_{f j}=S_{f j 0}+K_{f} \times \Delta P_{f} \quad(j=s L, n R) \\
S_{k}=S_{f k 0}-K_{f} \times \Delta P_{f} \quad(k=s R, n L)
\end{gathered}
$$

where $\angle P_{f}$ means pressure which acted on the jet of the feedback LPA. Mass flow rate $Q f o j$ and output pressure $P f_{o j}$ in outlet of the feedback LPA can be described by the following equations

$$
\begin{gathered}
Q_{f o j}=S_{f s j} \sqrt{2 \rho\left(P_{f s}-P_{f o j}\right)}-S_{f n j} \sqrt{2 \rho\left(P_{f o j}-P_{a t}\right)}-Q_{f l j} \\
\frac{d P_{f o j}}{d t}=\frac{\kappa R T_{f}}{V_{f o j}} Q_{f o j} \quad{ }_{(j=R, L)}
\end{gathered}
$$

We can know the static and dynamic characteristics of the optical on-off device by solving Eq. (1) to Eq. (14).

\section{CALCULATED RESULTS}

\section{Identification of optical on-off device}

In order to estimate and improve the performance of the tested valve by means of simulation, we identified

\begin{tabular}{|c|c|c|c|}
\hline$C_{d}$ & 0.1 & $T$ & $297[\mathrm{~K}]$ \\
\hline$K_{u}$ & $6.0 \times 10^{-11}\left[\mathrm{~m}^{2} / \mathrm{W}\right]$ & $\Delta P_{d R}, \Delta P_{d L}$ & $8.0[\mathrm{kPa}]$ \\
\hline$K_{f}$ & $1.15 \times 10^{-7}\left[\mathrm{~m}^{2} / \mathrm{Pa}\right]$ & $V_{i o R \theta}, V_{i o L O}$ & $3.46 \times 10^{-7}\left[\mathrm{~m}^{3}\right]$ \\
\hline$P_{a t}$ & $103[\mathrm{kPa}]$ & $V_{i o R O}, V_{i o L O}$ & $1.05 \times 10^{-6}\left[\mathrm{~m}^{3}\right]$ \\
\hline$P_{\text {is }}$ & $240[\mathrm{kPa}]$ & $V_{f o R \theta}, V_{f o L}$ & $2.28 \times 10^{-6}\left[\mathrm{~m}^{3}\right]$ \\
\hline$P_{f s}$ & $143[\mathrm{kPa}]$ & $\kappa$ & 1.4 \\
\hline$R$ & $287[\mathrm{~J} / \mathrm{kg} \cdot \mathrm{K}]$ & $\rho$ & $1.29\left[\mathrm{~kg} / \mathrm{m}^{3}\right]$ \\
\hline$S_{i n L \theta} S_{i s R O}$ & $3.86 \times 10^{-8}\left[\mathrm{~m}^{2}\right]$ & $S_{i o f R}$ & $4.00 \times 10^{-7}\left[\mathrm{~m}^{2}\right]$ \\
\hline$S_{\text {isLO }} S_{\text {inRo }}$ & $8.14 \times 10^{-8}\left[\mathrm{~m}^{2}\right]$ & $S_{\text {iog } L}$ & $5.50 \times 10^{-7}\left[\mathrm{~m}^{2}\right]$ \\
\hline$S_{f n L \theta} S_{f s R O}$ & $1.01 \times 10^{-7}\left[\mathrm{~m}^{2}\right]$ & $S_{f o R}$ & $1.20 \times 10^{-7}\left[\mathrm{~m}^{2}\right]$ \\
\hline$S_{f s L \theta} S_{f n R d}$ & $5.92 \times 10^{-7}\left[\mathrm{~m}^{2}\right]$ & $S_{f o L}$ & $5.00 \times 10^{-8}\left[\mathrm{~m}^{2}\right]$ \\
\hline
\end{tabular}
system parameters of the valve such as the area of opening of the interface and LPA. System parameters in the analytical model are identified as follows: Values of the initial areas of the opening in the photo-fluidic interface and LPAs are determined based on the dimension of the interface and LPAs so as to agree the calculated output pressure using the model with the
Table 1 Identified system parameters

static value of output pressure. The empirical constant $K u$ and $K f$ and area of opening in feedback flow passage are determined so as to agree the calculated results using Eq. (1) to Eq. (14) with step response of output pressure of the valve. The results, identified system parameters of the device, are shown in Table 1.

Comparison with experimental results

In order to confirm the validity of the proposed analytical model, we compare the results simulated using the analytical model and system parameters shown in Table 1, with experimental results for the optical on-off device. Figure 9 shows the transient response of output differential pressure of optical on-off device for stepwise input optical power of $25 \mathrm{~mW}$. In Fig.9, symbols $\bigcirc$ show experimental results, and the solid line shows theoretical results which are solved by the Runge-KuttaGill method. It can be seen that theoretical and experimental results agree well, and it is confirmed that the proposed analytical model of the valve is valid.

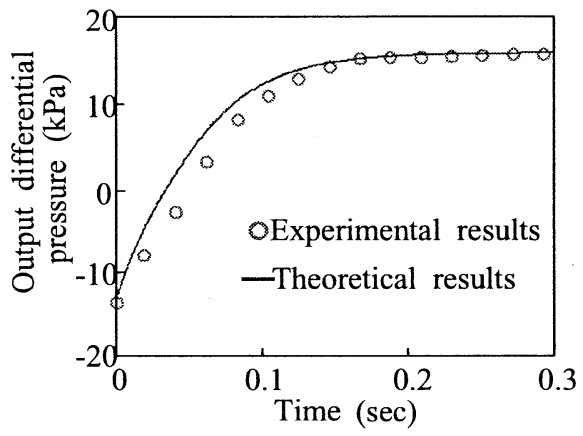

Fig.9 Comparison with experimental results

\section{IMPROVEMENT OF OPTICALON-OFF DEVICE}

\section{Improved optical on-off device}

The dynamic performance of the tested on-off valve depended on the dynamics of the optical on-off device. In order to improve the dynamics of the valve, we aim to decrease the volume and length of pipe connected with the device such as a feedback passage. Figure 10 shows construction of an improved optical on-off device. The device has a feedback passage and fluid resistance as a 


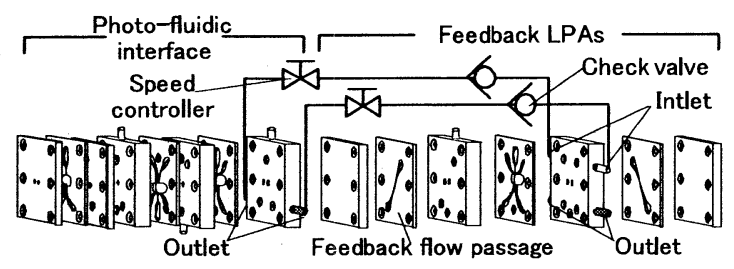

Fig.10 Construction of improved optical on-off device

laminate. In order to realize a suitable flow resistance, the sectional area of the flow passage in the laminate is decided by the identified parameters of the device based on the analytical model mentioned above. Each area of passages $S f o L$ and $S f o R$ is $0.05 \mathrm{~mm}^{2}$ and $0.15 \mathrm{~mm}^{2}$, respectively.

\section{Dynamic characteristics}

Figure 11 shows the transient response of output pressure of the improved device when stepwise optical power of $25 \mathrm{~mW}$ is applied. In Fig.11, each symbol $\bigcirc$ and $\triangle$ shows a result when optical signal switches on and off, respectively. From Fig.11, it can be seen that response time when the device switches on is $0.083 \mathrm{~s}$, and the response time when the device switches off is $0.094 \mathrm{~s}$. Compared with the response time of the previous type as shown in Table 2, we can confirm that the dynamics of the device is improved with the response time decreasing about $17 \%$. We think that it is possible to improve the dynamics by miniaturization and integration of other flow passages that have check valves and speed controllers.

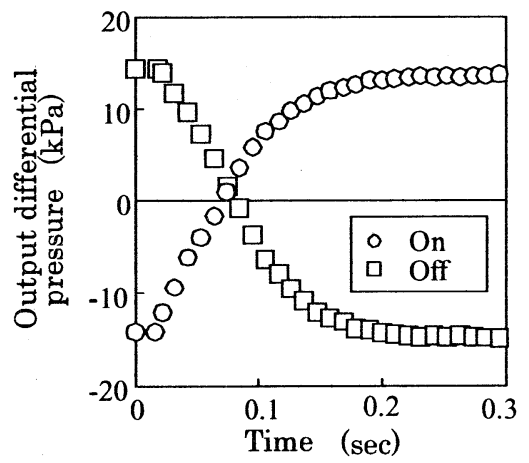

Fig.11 Step response of improved optical on-off device

Table 2 Comparison with old type

\begin{tabular}{l|c|c|c}
\hline \multirow{2}{\text{Sumof}}{\begin{tabular}{c||c|} 
Dead time and \\
Time constant \\
[sec]
\end{tabular}} & Input & Old type & Improved type \\
\cline { 2 - 4 } & Off & 0.098 & 0.083 \\
\hline \multicolumn{2}{l}{ Output pressure $[\mathrm{kPa}]$} & 0.115 & 0.094 \\
\hline
\end{tabular}

\section{CONCLUSIONS}

This study was done to develop the optical on-off valve and the study can be summarized as follows:
1) We proposed and tested an optical on-off device that consists of a photo-fluidic interface and feedback LPAs. And we investigated static and dynamic characteristics of the device. As a result, it can be found that the generated differential pressure of the device (about $\pm 14 \mathrm{kPa}$ ) is 9.3 times as much as that of the photo-fluidic interface. As a result of investigation of the step response, we found that the response time of the device is $0.098 \mathrm{~s}$ (on) and $0.115 \mathrm{~s}$ (off).

2) By using the optical on-off device and fluid amplifier, we constructed the an optical on-off valve. And we investigated static and dynamic characteristics of the device. As a result, it can be found that the generated differential pressure of the valve (about $\pm 155 \mathrm{kPa}$ ) is 4.5 times as much as that of the previous optical servo valve

3) We proposed an analytical model of the optical onoff device. And we identified the system parameters of the device. We confirmed the validity of the model and identified system parameters by comparing with experimental results. And we improved the dynamics of the device by using tested feedback flow passage plate whose sectional area of passage were determined by the analytical results.

We are going to improve the dynamics of the optical on-off valve by increasing the output flow rate and modifying a fluid booster amplifier in our future study. Finally, we express our thanks that a part of this research was supported by scientific research funding.

\section{REFERENCES}

1. T. Nakada, et al., Trans. of the JSME, 57-542(c), 1991,3228-3233. (in Japanese)

2. J. O. Gurney, Photo fluidic Interface, Trans. of the ASME, J. of Dyn. Syst., Meas., and Control, 1063,1984, 90-97.

3. S. Dohta, et al., A Study of Photo-Fluidic Interface, Proc. of 3rd FLUCOME'91, ASME, 1991, 193-197.

4. T. Akagi, et al., Trans. of the JSME, 62-600(c), 1996, 3188-3195. (in Japaese)

5. T. Akagi, et al., Analysis of an Optical Servo Valve and Improvement of an Opto-Pneumatic Control System, Proc.of 5th FLUCOME'97, 1997, 535-540.

6. T. Akagi, et al., Studies on Opto-Pneumatic Servo System, Trans. of the JSME, Int. J., 42-1(c), 1999, 171-179.

7. T. Akagi, et al., A Basic Study on Optimal Design of Opto-Pneumatic Servo System, Proc. of 4th JHPS Int. Symp. on Fluid Power, 1999, 425-430.

8. S. Dohta, et al., Optical Servo Valve and Its Optimal Design, Proc. of 6th FLUCOME'2000, Canada, 2000, FL-068. 